\title{
Moment Maps at the Quantum Level
}

\author{
Jiang-Hua Lu* \\ Department of Mathematics, MIT, Cambridge, MA 02139, USA
}

Received December 8, 1992; in revised form January 22, 1993

\begin{abstract}
We introduce the notion of moment maps for quantum groups acting on their module algebras. When the module algebras are quantizations of Poisson manifolds, we prove that the construction at the quantum level is a quantization of that at the semi-classical level. We also prove that the corresponding smashed product algebras are quantizations of the semi-direct product Poisson structures.
\end{abstract}

\section{Introduction}

The concept of moment maps for Hamiltonian actions is a very important one in symplectic geometry. In particular, it is a crucial tool in the study of symplectic reduced spaces, on which the reduced Hamiltonian systems live [Ms-We]. In the theory of Hopf algebras, a very important notion is that of inner actions (see [B-C-M] and the references therein). In the first part of this paper, we relate these two concepts in the two different fields. We show that by taking semi-classical limits, inner actions of Hopf algebras give rise to Poisson actions of Poisson groups with moment maps. This leads to the definition of moment maps for quantum group actions, the first step in carrying out quantum reduction.

An equally important concept in Hopf algebra theory is that of crossed products [B-C-M]. We show that in symplectic and Poisson geometry, this corresponds to semi-direct products of Poisson manifolds and Poisson groups.

Recall that if $P$ is a symplectic manifold equipped with an action of a group $G$ preserving the symplectic structure, then the space of $G$-invariant functions on $P$ is closed under the Poisson bracket on functions on $P$, and thus the quotient space $P / G$, when it is a manifold, has a naturally defined Poisson structure. Flows of $G$-invariant Hamiltonians can be considered as living on $P / G$. More precisely, they live on the symplectic leaves in $P / G$. When the action is generated by a moment map $\phi: P \rightarrow \mathrm{g}^{*}$, where $\mathrm{g}$ is the Lie algebra of $G$, the reduction procedure

* Research partially supported by NSF grant DMS-89-07710 
of Marsden-Weinstein [Ms-We] gives a way of describing symplectic leaves in $P / G$ as the quotients $\phi^{-1}(\mathcal{O}) / G$, where $\mathcal{O}$ is a co-adjoint orbit in $\mathrm{g}^{*}$. This description gives a one-one correspondence between symplectic leaves in $P / G$ and co-adjoint orbits in $\mathrm{g}^{*}$. actions.

In the theory of quantum spaces, symmetries are provided by quantum group

Definition 1.1. Let $A$ be a Hopf algebra. By a left $A$-module algebra we mean a left $A$-module $V$ which is an algebra at the same time and the action of $A$ on $V$ respects the algebra structure on $V$ in the following sense:

1) $a \cdot 1_{\mathrm{v}}=\varepsilon(a) 1_{\mathrm{v}}$ for all $a \in A$,

2) $a \cdot(v u)=\left(a_{(1)} \cdot v\right)\left(a_{(2)} \cdot u\right)$ for all $a \in A$ and $v, u \in V$, where $1_{\mathrm{A}}$ is the unit element of $A, 1_{\mathrm{v}}$ is the unit element in $V$, and $\Delta a=\sum a_{(1)} \otimes a_{(2)}$ is the co-product of $a$.

In [Sw], $A$ is said to "measure" $V$ to $V$ if these two conditions are satisfied. Condition 1) says that the map $k \rightarrow V: \alpha \mapsto \alpha 1_{\mathrm{v}}$ is an $A$-module map. Condition 2) says that the multiplication map $m_{\mathrm{v}}: V \otimes V \rightarrow V$ is an $A$-module map, where $A$ acts on $V \otimes V$ via the co-product map $\Delta$.

Semi-classical limits of quantum groups are the so-called Poisson groups, and semi-classical limits of quantum group actions on module algebras are Poisson actions of Poisson groups on Poisson manifolds (see Sect. 2 for the definitions). The theory of moment maps and reduction for Poisson actions has been studied in [Lu1]. In particular, a moment map for a Poisson action of $G$ on $P$ is a Poisson map from $P$ to the dual Poisson group $G^{*}$ of $G$. For an action of $G$ on $P$ with such a moment map, there is again the Marsden-Weinstein reduction procedure that constructs symplectic leaves of the quotient space $P / G$ from symplectic leaves in $G^{*}$.

Let $A$ be a Hopf algebra and let $A^{*}$ be its dual Hopf algebra. If $A$ is a quantization of a Poisson group $G$ (see the definition in Sect. 2), then according to the Quantum Duality Principle in [STS2], $A^{*} \cong U_{h} \mathrm{~g}$ is a quantization of the dual group $G^{*}$, and $A^{*}$-module algebras are quantum analogs of Poisson actions of $G$. Now suppose $V$ is a left $A^{*}$-module algebra. It is easy to show (see Sect. 3) that the space of $A^{*}$-invariant elements in $V$ form a subalgebra $V^{A^{*}}$ of $V$. If we think of $V$ as quantizing a symplectic manifold $P$ (see definition in Sect. 2), then the algebra $V^{A^{*}}$ should be considered as quantizing the manifold $P / G$. If we take the general principle of the "orbit method" that symplectic leaves of a Poisson manifold correspond to irreducible representations of its quantized algebra, it is natural to want to describe irreducible representations of the algebra $V^{A^{*}}$ from that of $A^{*}$. It is here where we need the notion of moment maps at the quantum level. actions.

In this paper, we propose the following definition of moment maps for quantum

Definition 1.2. Let $D: A^{*} \otimes V \rightarrow V$ be an action of $A^{*}$ on $V$ making $V$ into a left $A^{*}$-module. An algebra homomorphism $\Phi: A^{*} \rightarrow V$ is called a moment map for $D$ if

$$
D(x \otimes v)=\Phi\left(x_{(1)}\right) v \Phi\left(S\left(x_{(2)}\right)\right),
$$

where $\Delta x=x_{(1)} \otimes x_{(2)}$ denotes the co-product of $x$, and $S$ denotes the antipode map of $A^{*}$.

It is well-known in Hopf algebra theory [B-C-M] that an algebra homomorphism from $A^{*}$ to $V$ always defines a left $A^{*}$-module structure on $V$ by the above 
formula. In this paper, we show that this is the correct notion of moment maps for quantum actions. We do this by showing that when $A$ is a quantum group and when $V$ is a quantization of a symplectic (or Poisson) manifold, the "semi-classical limit of this concept" coincides with the one for Poisson group actions given in [Lu1]. This is the content of Theorem 3.10.

Once we have the correct notion of moment maps, the next step will be to find a quantum analog of the Marsden-Weinstein procedure for quantum actions with moment maps. This amounts to relating irreducible representations of the algebra $V^{A^{*}}$ to that of the algebra $A^{*}$. It is easy to show that the two subalgebras $V^{A^{*}}$ and $\Phi\left(A^{*}\right)$ of $V$ commute with each other in $V$. In fact, the subalgebra $V^{A^{*}}$ is the centralizer of $\Phi\left(A^{*}\right)$ in $V$. If $\Phi\left(A^{*}\right)$ is also the centralizer of $V^{A^{*}}$ in $V$, then we are in the situation of a "Howe Pair" [Ho]. Recall that the principle for relating irreducible representations of the two subalgebras in a "Howe pair" situation is to decompose a "good" irreducible representation of $V$ with respect to the tensor product of the two subalgebras. Each irreducible component in this decomposition will be a tensor product of irreducible representations of the two subalgebras, and this, in good cases, will enable us to get a one-one correspondence between all irreducible representations of the two subalgebras. This principle has only been checked for special cases. For this reason, we do not think that one can formulate a general quantum analog of the Marsden-Weinstein reduction procedure. Instead, one can try to work with concrete examples. We hope to be able to do this when we study quantum groupoids.

It is typical in quantum group theory that subgroups at the classical level do not correspond to quantum subgroups. At the semi-classical level, they are the so-called coisotropic subgroups [Lu1]. A general question is: if a Poisson manifold $P$ is quantized to an algebra $V$, what should coisotropic submanifolds in $P$ correspond to in $V$ ? An answer to this question is needed when studying quantum groupoids. We answer this question by proposing the following "Poisson creed" (compare to the "symplectic creed" stated in [We1] by Weinstein).

Creed. Coisotropic submanifolds of $P$ correspond to one-sided ideals in $V$.

In Sect. 3, we give examples where this is verified. The quantum analog of a coisotropic subgroup of a Poisson group is then a subspace of a Hopf algebra which is a one-sided ideal with respect to the product and a co-ideal with respect to the co-product. We describe reductions with respect to such "one-sided Hopf ideals" in Sect. 3.

The last part of the paper deals with smash products, where moment maps appear naturally. It is well-known in Hopf algebra theory [Sw] that if $V$ is a left $A^{*}$-module algebra, then there is a naturally defined algebra structure on $V \otimes A^{*}$, the so-called smash product or semi-direct product or cross-product, which encodes the algebra structures on $V$ and $A^{*}$ as well as the action of $A^{*}$ on $V$. We show that when $A$ is a quantum group and when $V$ is a quantization of a Poisson manifold, the semi-classical limit of the smash product algebra is the so-called semi-direct product Poisson structure as defined in [Lu1]. As a special example, if we take the left regular action of $A^{*}$ on $A$, the resulting smash product structure on $A \otimes A^{*}$ is called the Heisenberg double of $A$ in [STS2], which quantizes a canonical symplectic structure on $G \times G^{*}$. This symplectic structure is compatible with a natural groupoid structure on $G \times G^{*}$ over $G$ [Lu-We2]. This is our main motivating example of quantum groupoids. 


\section{Semi-Classical Limits}

Recall that a Poisson algebra is a commutative algebra $A$ together with a Lie bracket \{\} , also called a Poisson bracket, such that

$$
\{a b, c\}=a\{b, c\}+b\{a, c\}, \quad a, b, c \in A .
$$

A Poisson manifold is a manifold $P$ together with a Poisson algebra structure on the algebra Fun $(P)$ of functions on $P$. Here functions on $P$ are understood to be $C^{\infty}$ or algebraic depending on whether $P$ is $C^{\infty}$ or algebraic. A Poisson map from a Poisson manifold to another is a map whose pull-back on functions is a Lie algebra homomorphism with respect to the Poisson brackets.

Definition 2.1. By a quantization of the Poisson structure \{\} on $P$ we mean a one parameter family of non-commutative algebra structures, denoted by $*_{h}$, on the vector space Fun $(P)$ such that

1) $*_{0}$ corresponds to the commutative multiplication, and

2) the "derivative of $*_{h}$ at $h=0$ " is the Poisson bracket, i.e.,

for $f, g \in \operatorname{Fun}(P)$.

$$
\{f, g\}=\lim _{h \rightarrow 0} \frac{1}{h}\left(f *_{h} g-g *_{h} f\right)
$$

We note that any one parameter family of non-commutative algebra structures $*_{h}$ on Fun $(P)$ which satisfies 1$)$ in the above definition will define a Poisson bracket \{\} on $P$ given by the formula in 2). This Poisson bracket is called the semi-classical limit of $*_{h}$.

Definition 2.2. By a quantum group we mean a Hopf algebra $\left(\operatorname{Fun}(G), *_{h}, \Delta, S_{h}, \varepsilon_{h}\right)$ consisting of

1) a one parameter family of associative algebra structures $*_{h}$ on the space of functions $\operatorname{Fun}(G)$ of a group $G$ (here $G$ can be $C^{\infty}$, algebraic or formal);

2) the map

$$
\Delta: \operatorname{Fun}(G) \rightarrow \operatorname{Fun}(G) \otimes \operatorname{Fun}(G): \Delta(f)\left(g_{1}, g_{2}\right)=f\left(g_{1} g_{2}\right), \quad g_{1}, g_{2} \in G,
$$

which is the pull-back of the group multiplication map of $G$;

3) (the antipode) a map $S_{h}$ from $\operatorname{Fun}(G)$ to itself such that $S_{0}$ is given by $S_{0}(f)(g)=f\left(g^{-1}\right) ;$ and

4) (the co-unit) a map $\varepsilon_{h}$ from $\operatorname{Fun}(G)$ to the ground field $k$ such that $\varepsilon_{0}$ is given by $\varepsilon_{0}(f)=f(e)$, where $e \in G$ is the unit element of $G$.

We emphasize here that in our definition of a quantum group the co-product $\Delta$ is "not quantized" in the sense that it is simply the pull-back on functions of the group multiplication map. In general, If $P$ and $Q$ are two Poisson manifolds and if $\left(\operatorname{Fun}(P), *_{h}\right)$ and $\left(\operatorname{Fun}(Q), *_{h}\right)$ are quantizations of $P$ and $Q$ respectively, we say that a Poisson map $\phi: P \rightarrow Q$ is compatible with the two quantizations if the map

$$
\phi^{*}: \operatorname{Fun}(Q) \rightarrow \operatorname{Fun}(P): f \mapsto f \circ \phi, f \in \operatorname{Fun}(Q),
$$

which is the pull-back of $\phi$ on functions, is an algebra homomorphism from $\left(\operatorname{Fun}(Q), *_{h}\right)$ to $\left(\operatorname{Fun}(P), *_{h}\right)$. For example, the identity map from $P$ to itself is compatible with any quantization of $P$. 
If $\left(\operatorname{Fun}(G), *_{h}, \Delta, S_{h}, \varepsilon_{h}\right)$ is a quantum group, the semi-classical limit of $*_{h}$ is then a Poisson structure on $G$ with the property that the group multiplication map

$$
G \times G \rightarrow G:(g, h) \mapsto g h
$$

is a Poisson map, where $G \times G$ is equipped with the direct product Poisson structure. Groups with such Poisson structures are called Poisson groups. We say that the quantum group $\left(\operatorname{Fun}(G), *_{h}, \Delta, S_{h}, \varepsilon_{h}\right)$ is a quantization of the Poisson group $G$. For simplicity, we will simply use $\operatorname{Fun}_{h}(G)$ to denote the Hopf algebra $\left(\operatorname{Fun}(G), *_{h}, \Delta, S_{h}, \varepsilon_{h}\right)$.

Definition 2.3. Let $A$ be a Hopf algebra. By a right $A$-co-module algebra, we mean a right $A$-co-module $V$ which is an algebra at the same time such that

1) the co-module map

$$
\sigma: V \rightarrow V \otimes A
$$

is an algebra homomorphism, where $V \otimes A$ is equipped with the tensor product algebra structure, and

2) $\left(\mathrm{id}_{\mathrm{v}} \otimes \varepsilon\right) \circ \sigma=\mathrm{id}_{\mathrm{v}}$.

Let $A$ be a quantum group quantizing a Poisson group $G$. Let $V$ be a quantization of a Poisson manifold $P$. Assume that $\sigma: V \rightarrow V \otimes A$ defines a right $A$ co-module structure on $V$. If the map $\sigma$ coincides with the pull-back on functions of some map $\sigma_{0}: P \times G \rightarrow P$, then $\sigma_{0}$ defines a right action of $G$ on $P$ and it is a Poisson map with the direct Poisson structure on $G \times P$. Actions of $G$ with this property are called Poisson actions. In this case, we say that the $A$-co-action on $V$ is a quantization of the Poisson action $\sigma_{0}$.

According to this definition, the right $A$-comodule structure on a quantum group $A$ itself given by the co-product of $A$ is a quantization of right action of the corresponding Poisson group on itself by right translations. Other examples are the vector co-representations of the quantum groups $A=\operatorname{fun}_{q}(G)$, where $G$ is a complex semi-simple Lie group of type $A_{n}, B_{n}, C_{n}$ or $D_{n}$, as are given in [R-T-F].

We now recall the theory of moment maps and reduction of Poisson actions [Lu1]. Their quantum counterparts will be discussed in Sect. 3.

One way of generating Poisson actions of Poisson groups is through Poisson maps to their dual groups. Let $P$ be a Poisson manifold and $\phi: P \rightarrow G^{*}$ a Poisson map. For each $X \in \mathfrak{g}$, let $X^{l}$ (resp. $X^{r}$ ) be the left (resp. right) invariant 1-forms on $G^{*}$ whose value at $e$ is $X$. Define a vector field $\sigma_{\mathrm{x}}\left(\right.$ resp. $\left.\sigma_{\mathrm{x}}^{\prime}\right)$ on $P$ by

$$
\sigma_{\mathrm{x}}=\pi_{\mathrm{P}}^{\#}\left(\phi^{*} X^{l}\right) \quad\left(\text { resp. } \sigma_{\mathrm{x}}^{\prime}=-\pi_{\mathrm{P}}^{\#}\left(\phi^{*} X^{r}\right)\right) \text {, }
$$

where $\pi_{\mathrm{P}}^{\#}$ is the bundle map $T^{*} P \rightarrow T P: \pi(\xi, \eta)=\xi\left(\pi_{\mathrm{P}}^{\#} \eta\right)$. Then the map $X \mapsto \sigma_{\mathrm{x}}$ (resp. the map $X \mapsto \sigma_{\mathrm{x}}^{\prime}$ ) defines a Lie algebra anti-homomorphism (resp. homomorphism) from $g$ to the Lie algebra of vector fields on $P$ with the commutator bracket. Assume that it can be integrated to an action of $G$ on $P$. It is then a left (resp. right) Poisson action of $G$ on $P$. See [Lu1] for more details.

Definition 2.4. A left (resp. right) Poisson action of $G$ on $P$ is said to have a moment map $\phi: P \rightarrow G^{*}$ if it is generated by $\phi$ as described above.

Definition 2.5. The left and right actions of $G$ on $G^{*}$ generated by the identity map $G^{*} \rightarrow G^{*}$ are respectively called the left and right dressing actions of $G$ on $G^{*}$. 
Similarly, the left and right dressing actions of $G^{*}$ on $G$ are generated by the identity map of $G$.

Recall that symplectic leaves of a Poisson manifold $\left(P, \pi_{\mathrm{P}}\right)$ are defined to be the integral submanifolds of the distribution defined by the image of the bundle map $\pi_{\mathrm{P}}^{\#}$. It is clear from the definition that

1) a Poisson action on $P$ with a moment map necessarily leaves the symplectic leaves in $P$ invariant. Consequently, the left action of $G$ on itself by left translations can not have a moment map;

2) the dressing oribits of $G^{*}$ in $G$ coincide with its symplectic leaves of $G$.

3) the Poisson map $\phi: P \rightarrow G^{*}$ is $G$-equivariant with respect the left (resp. right) action $\sigma$ (resp. $\sigma^{\prime}$ ) of $G$ on $P$ induced by $\phi$ and the left (resp. right) dressing action of $G$ on $G^{*}$.

Let $P \times G \rightarrow P$ be a Poisson action of $G$ on $P$. Then it is easy to show that the space of $G$-invariant functions on $P$ is invariant with respect to the Poisson bracket on functions on $P$. Therefore when the quotient space $P / G$ is a manifold, it inherits a Poisson structure such that the projection map $P \rightarrow P / G$ is a Poisson map. This procedure is usually referred to as Poisson reduction.

Sometimes we need reduction by subgroups of $G$. For example, given a right Poisson action of $G$ on $P$ and a left Poisson action of $G$ on $Q$, the diagonal action of $G$ on $P \times Q$ given by $g:(p, q) \mapsto\left(p \cdot g, g^{-1} \cdot q\right)$ is in general not a Poisson action, so the Poisson reduction procedure does not apply to the space $(P \times Q) / G$. But the group $G \times G$ acts naturally on $P \times Q$ from the right, and it is a Poisson action if we equip the second factor $G$ with minus the Poisson structure on $G$. We denote this Poisson group by $G \times \bar{G}$. We can think of $G$ as sitting inside $G \times \bar{G}$ as the diagonal. As such, it is a coisotropic subgroup of $G \times \bar{G}$.

In general, a subgroup $H$ of a Poisson group $G$ is called a coisotropic subgroup if as a submanifold of $G$ it is coisotropic, i.e., if the space of functions on $G$ that vanish on $H$ is closed under the Poisson bracket on $G$. The Poisson reduction procedure described above can also be carried out with respect to coisotropic subgroups of $G$, namely, if $P \times G \rightarrow P$ is a Poisson action of $G$ on $P$ and if $H$ is a coisotropic subgroup of $G$, then the space of $H$-invariant functions on $P$ is closed under the Poisson bracket on $P$. Such a procedure is usually referred to as reduction by coisotropic subgroups. In Sect. 3 , we will give a quantum analog of it.

\section{Moment Maps and Reduction at the Quantum Level}

In this section, we assume that $A$ is a Hopf algebra and $A^{*}$ is its dual Hopf algebra. The pairing $\left\langle>\right.$ between $A$ and $A^{*}$ then has the following properties:

$$
\langle a b, x\rangle=\left\langle a \otimes b, x_{(1)} \otimes x_{(2)}\right\rangle, \quad\langle a, x y\rangle=\left\langle a_{(1)} \otimes a_{(2)}, x \otimes y\right\rangle
$$

and

$$
\left\langle 1_{\mathrm{A}}, x\right\rangle=\varepsilon(x), \quad\left\langle a, 1_{\mathrm{A}^{*}}\right\rangle=\varepsilon(a), \quad\langle a, S(x)\rangle=\langle S(a), x\rangle,
$$

where $a, b \in A, x, y \in A^{*}$, and we are using the simplified notation $a_{(1)} \otimes a_{(2)}$ to denote the element $\Delta(a)=\sum a_{(1)} \otimes a_{(2)}$.

Although the theory in this section is true for general Hopf algebras, we will think of $A$ as a quantum group quantizing a Poisson group $G$ and think of $A^{*}$ as 
quantizing the dual group $G^{*}$ of $G$. Similarly, $A$-co-module algebras or $A^{*}$-module algebras should be thought of as quantizations of some Poisson manifolds on which the Poisson group $G$ acts in a Poisson fashion. This enables us to use the concepts and constructions at the semi-classical level to motivate those at the quantum level.

We first look at the quantum analog of Poisson reduction.

Let $V$ be an $A^{*}$-module algebra. An element $v$ of $V$ is said to be $A^{*}$-invariant if

$$
x \cdot v=\varepsilon(x) v, \quad \forall x \in A^{*} .
$$

Set

$$
V^{A^{*}}=\left\{v \in V: v \text { is } A^{*} \text {-invariant }\right\} .
$$

The proof of the following proposition follows directly from the definitions.

Proposition 3.1. $V^{A^{*}}$ is a subalgebra of $V$ containing the unit element $1_{\mathrm{v}}$.

We now discuss the quantum analog of reduction by coisotropic subgroups as discussed in Sect. 2. To do this, we have to answer the following question first:

Question 3.2. If $G$ is a Poisson group and $A=\operatorname{Fun}_{h}(G)$ is a quantization of $G$, what do coisotropic subgroups of $G$ correspond to in $A$ ?

More generally,

Question 3.3. Suppose that $A_{\mathrm{P}}$ is a quantization of the Poisson maniflold $P$, what should coisotropic submanifolds of $P$ correspond to in $A_{\mathrm{P}}$ ?

Semi-classically, a coisotropic submanifold $P_{1}$ of $P$ can be described by the subspace of Fun $(P)$ consisting of functions that vanish on $P_{1}$. It is an ideal with respect to the commutative multiplication and a subalgebra with respect to the Poisson bracket. Its quantum counterpart in $A_{\mathrm{P}}$ should be "more than" just a subalgebra since the classical limit of a subalgebra would only be a subalgebra with respect to the commutative multiplication. It should also be "less than" a (two-sided) ideal since the semi-classical limit of a two-sided ideal would be an ideal with respect to the Poisson bracket. A natural candidate is then a one-sided ideal in $A_{\mathrm{P}}$. On the other hand, one-sided ideals in $A_{\mathrm{P}}$ indeed have the correct classical and semi-classical limits. We state this in the form of the following "creed" (compare to the "sympletic creed" stated in [We1] by Weinstein).

Creed. Coisotropic submanifolds of $P$ correspond to one-sided ideals in $A_{\mathrm{P}}$.

We now give one example of this correspondence.

Example 3.4. Let $\left(P_{1},\{\}_{1}\right)$ and $\left(P_{2},\{\}_{2}\right)$ be two Poisson manifolds, and let $\phi$ : $P_{2} \rightarrow P_{1}$ be a Poisson map. Then the graph $\Gamma$ of $\phi$, described by the embedding of $P_{2}$ into $P_{1} \times P_{2}$ :

$$
P_{2} \rightarrow P_{1} \times P_{2}: x \mapsto(\phi(x), x),
$$

is a coisotropic submanifold of $P_{1} \times P_{2}^{-}$[We2], where $P_{2}^{-}$means the manifold $P_{2}$ equipped with the Poisson structure -\{\}$_{2}$. The above embedding induces a map

$$
\lambda: C^{\infty}\left(P_{1}\right) \otimes C^{\infty}\left(P_{2}\right) \rightarrow C^{\infty}\left(P_{2}\right): f_{1} \otimes f_{2} \mapsto\left(f_{1} \circ \phi\right) f_{2}
$$

and the space of functions on $P_{1} \times P_{2}$ that vanish on the graph $\Gamma$, is clearly the kernel of the map $\lambda$. Assume now that $A_{1}$ and $A_{2}$ are quantizations of $\left(P_{1},\{\}_{1}\right)$ and 
$\left(P_{2},\{\}_{2}\right)$ respectively. Assume also that the Poisson map $\phi$, or rather, the Poisson algebra homomorphism $C^{\infty}\left(P_{1}\right) \rightarrow C^{\infty}\left(P_{2}\right): f_{1} \mapsto f_{1} \circ \phi$, is quantized to an algebra homomorphism $\Phi: A_{1} \rightarrow A_{2}$. Then the quantum counterpart of $\lambda$ is the map

$$
\Lambda: A_{1} \otimes A_{2}^{\text {op }} \rightarrow A_{2}: a_{1} \otimes a_{2} \mapsto \Phi\left(a_{1}\right) a_{2},
$$

where $A_{2}^{\text {op }}$ is the space $A_{2}$ equipped with the algebra structure opposite to that of $A_{2}$, and the kernel $I$ of the map $\Lambda$ should clearly be the quantum analog of the coisotropic submanifold $\Gamma$ in $A_{1} \otimes A_{2}^{\text {op }}$. It is easy to check that $I$ is a left ideal of $A_{1} \otimes A_{2}^{\mathrm{op}}$.

Returning to Question 3.2, it is now clear that a coisotropic subgroup of $G$ should correspond to a subspace of $A$ which is a one-sided ideal with respect to the product and a co-ideal with respect to the co-product. We propose to call such subspaces one-sided Hopf ideals. The author would like to thank Y. Soibelman for suggesting this name.

Proposition 3.5. Suppose that $I \subset A$ is a left Hopf ideal. Set

$$
I^{\perp}=\left\{x \in A^{*}:\langle x, a\rangle=0 \forall a \in I\right\} \subset A^{*} .
$$

The $I^{\perp}$ is a left coideal subalgebra of $A^{*}$, i.e., it is a subalgebra of $A^{*}$ and $\Delta I^{\perp} \subset A^{*} \otimes I^{\perp}$.

Proof. Let $x, y \in I^{\perp}$. Then for any $a \in I$, since $\Delta a \in A^{*} \otimes I+I \otimes A^{*}$, we have

$$
\langle x y, a\rangle=\langle x \otimes y, \Delta a\rangle=0 .
$$

Hence $x y \in I^{\perp}$ and $I^{\perp}$ is a subalgebra of $A^{*}$. Let $x \in I^{\perp}$. Then for any $a \in A, b \in I$, we have

$$
\langle\Delta x, a \otimes b\rangle=\langle x, a b\rangle .
$$

Since $I$ is a left ideal, $a b \in I$. Hence

$$
\langle\Delta x, a \otimes b\rangle=0 .
$$

It follows that $\Delta x \in A^{*} \otimes I^{\perp}$.

Q.E.D.

We now have the following reduction by one-sided coideal subalgebras. It is the quantum analog of reduction by coisotropic subgroups.

Proposition 3.6. Let $V$ be an $A^{*}$-module algebra. Let $J \subset A^{*}$ be a left co-ideal subalgebra of $A^{*}$. Set

$$
V^{J}=\{v \in V: x \cdot v=\varepsilon(x) v \forall x \in J\} .
$$

Then $V^{J}$ is a subalgebra of $V$ containing the unit element $1_{\mathrm{v}}$.

Proof. That $V^{J}$ contains the unit element follows directly from the definition. Let $v, u \in V^{J}$. Then for any $x \in J$, we have

$$
x \cdot(v u)=\left(x_{(1)} \cdot v\right)\left(x_{(2)} \cdot u\right),
$$

where $\Delta x=\sum x_{(1)} \otimes x_{(2)}$ and is written as $x_{(1)} \otimes x_{(2)}$ for simplicity. From Proposition 3.5, $\Delta x \in A^{*} \otimes J$, so $x_{(2)} \in J$. Thus $x_{(2)} \cdot u=\varepsilon\left(x_{(2)}\right) u$. Therefore

$$
\begin{aligned}
x \cdot(v u) & =\left(x_{(1)} \cdot v\right) \varepsilon\left(x_{(2)}\right) u \\
& =\left(\varepsilon\left(x_{(2)}\right) x_{(1)} \cdot v\right) u .
\end{aligned}
$$


But $\varepsilon\left(x_{(2)}\right) x_{(1)}=x$. Hence

$$
x \cdot(v u)=(x \cdot v) u=\varepsilon(x) v u .
$$

Hence $v u \in V^{J}$. This shows that $V^{J}$ is a subalgebra of $V$.

Example 3.7. If $V$ and $W$ are two left $A^{*}$-module algebras, the $V \otimes W$ is a left $A^{*} \otimes A^{*}$-module algebra. Reduction of $V \otimes W$ with respect to the "diagonal" $J:=\Delta\left(A^{*}\right)$ of $A^{*} \otimes A^{*}$ is then a quantum analog of reduction by the diagonal subgroups in the classical and semi-classical cases.

We now turn to the study of quantum moment maps. Recall that a Poisson map $\phi: P \rightarrow G^{*}$ from a Poisson manifold $P$ to the dual group $G^{*}$ of a Poisson group $G$ induces a left (or right) Poisson action of $G$ on $P$ by Formula (2), and the map $\phi$ is called its moment map.

Analogously, we have the following well-known fact in Hopf algebra theory (see, for example, [B-C-M, Mj]).

Proposition 3.8. If $V$ is an algebra and if $\Phi: A^{*} \rightarrow V$ is an algebra homopmorphism, then the following map

$$
D^{\Phi}: A^{*} \otimes V \rightarrow V: x \otimes v \mapsto x(v):=\Phi\left(x_{(1)}\right) v \Phi\left(S\left(x_{(2)}\right)\right),
$$

where $\Delta x=x_{(1)} \otimes x_{(2)}$, defines a left action of $A^{*}$ on $V$ and it makes $V$ into a left $A^{*}$-module algebra.

Definition 3.9. The map $\Phi: A^{*} \rightarrow V$ is called the moment map for the action $D^{\Phi}$ of $A^{*}$ on $V$ given by Formula (4).

Similarly, one can define a right action of $A^{*}$ on $V$ by

$$
V \otimes A^{*} \rightarrow V: v \otimes x \mapsto \Phi\left(S\left(x_{(1)}\right)\right) v \Phi\left(x_{(2)}\right),
$$

and $\Phi: A^{*} \rightarrow V$ is called its moment map.

To justify the name "moment map" for the map $\Phi$, we now study the semiclassical limit of the action $D^{\Phi}$. To this end, we assume that $A^{*}$ is a quantization of $G^{*}$, the dual group of a Poisson group $G$. Assume also that $V$ is a quantization of the Poisson manifold $P$, and that the map $\Phi: A^{*} \rightarrow V$ coincides with the pull-back of a map $\phi: P \rightarrow G^{*}$. Then the fact that $\Phi$ is an algebra homomorphism implies that $\phi$ is a Poisson map. We have

$$
\begin{aligned}
\lim _{h \rightarrow 0} \frac{1}{h}(x(v)-\varepsilon(x) v) & =\lim _{h \rightarrow 0} \frac{1}{h}\left(\Phi\left(x_{(1)}\right) v \Phi\left(S\left(x_{(2)}\right)\right)-v \Phi\left(x_{(1)}\right) \Phi\left(S\left(x_{(2)}\right)\right)\right) \\
& =\lim _{h \rightarrow 0}\left(\Phi\left(x_{(1)}\right) v-v \Phi\left(x_{(1)}\right)\right) \Phi\left(S\left(x_{(2)}\right)\right) \\
& =\left\{\phi^{*} x_{(1)}, v\right\} \phi^{*}\left(S\left(x_{(2)}\right)\right) .
\end{aligned}
$$

Here for simplicity, we are omitting the $*_{h}$-symbols in the first two identities, and we are using $S$ to denote both $S_{h}$ and $S_{0}$. The multiplication in the last identity is the commutative one on Fun $(P)$. Denote by $\sigma_{x}$ the vector field on $P$ given by

$$
\operatorname{Fun}(P) \ni v \mapsto\left\{\phi^{*} x_{(1)}, v\right\} \phi^{*}\left(S\left(x_{(2)}\right)\right),
$$

or

$$
\sigma_{x}=-\phi^{*}\left(S\left(x_{(2)}\right)\right) H_{\phi^{*} x_{(1)}}
$$


where for $u \in \operatorname{Fun}(P), H_{u}: v \mapsto\{v, u\}$ denotes the Hamiltonian vector field of $u$. Therefore each $x \in \operatorname{Fun}\left(G^{*}\right)$ defines a vector field $\sigma_{x}$ on $P$. The fact that $D^{\Phi}$ is a left action of $A^{*}$ on $V$ implies that the map $x \mapsto \sigma_{x}$ is a Lie algebra homomorphism from $\left(\operatorname{Fun}\left(G^{*}\right),\{\}\right)$ to the Lie algebra of vector fields on $P$ with the commutator bracket.

Theorem 3.10. The infinitesimal action $x \mapsto \sigma_{x}$ of $\left(\mathrm{Fun}\left(G^{*}\right),\{\}\right)$ on $P$ is the same as the right infinitesimal action $X \mapsto \sigma_{X}^{\prime}$ of $\mathrm{g}$ on $P$ induced by the Poisson map $\phi$ : $P \rightarrow G^{*}$ as given by Formula (2). Therefore, the action $D^{\Phi}$ of $A^{*}$ on $V$ is a quantization of the Poisson action of $G$ on $P$ induced by $\phi$.

Proof. For $x \in \operatorname{Fun}\left(G^{*}\right)$, consider the 1 -form $\theta_{x}$ on $G^{*}$ given by

$$
\theta_{x}=S\left(x_{(2)}\right) d x_{(1)} \text {. }
$$

Clearly, the vector field $\sigma_{x}$ on $P$ is given by

$$
\sigma_{x}=-\pi_{\mathrm{P}}^{\#} \phi^{*}\left(\theta_{x}\right) \text {. }
$$

Comparing with Formula (2) for the vector field $\sigma_{X}^{\prime}$, where $X \in \mathfrak{g}$, we see that it remains to show that the 1 -form $\theta_{x}$ is right invariant. This is indeed so, for let $\xi \in \mathfrak{g}^{*}$ and $g \in G^{*}$, then we have

$$
\begin{aligned}
\left\langle r_{g}^{*} \theta_{x}(g), \xi\right\rangle & =\left.\frac{d}{d t}\right|_{t=0} S\left(x_{(2)}\right)(g) x_{(1)}(\exp t \xi \cdot g) \\
& =\left.\frac{d}{d t}\right|_{t=0} x_{(1)}(\exp t \xi \cdot g) x_{(2)}\left(g^{-1}\right) \\
& =\left.\frac{d}{d t}\right|_{t=0} x\left(\exp t \xi \cdot g \cdot g^{-1}\right) \\
& =\langle d x(e), \xi\rangle .
\end{aligned}
$$

This shows that the 1 -form $\theta_{x}$ on $G^{*}$ is right invariant and that its value at the identity element $e$ of $G^{*}$ is equal to $d x(e)$. Let $X=d x(e) \in \mathfrak{g}^{*}$. Then $\sigma_{x}=\sigma_{X}^{\prime}$. This correspondence allows us to say that the infinitesimal action of $(\operatorname{Fun}(P),\{\})$ on $P$ is the same as that of $\mathfrak{g}$ on $P$ given by $X \mapsto \sigma_{X}^{\prime}$.

Q.E.D.

Having seen the justification for the map $\Phi: A^{*} \rightarrow V$ to be called the moment map for the action $D^{\Phi}$, we now study some properties of $D^{\Phi}$ and $\Phi$, which, again, are obvious quantum analogs of those at the semi-classical level.

Definition 3.11. The left (resp. right) action of $A^{*}$ on itself induced by the identity


given by

$$
\begin{gathered}
\operatorname{ad}^{l}: A^{*} \otimes A^{*} \rightarrow A^{*}: x \otimes y \mapsto x_{(1)} y S\left(x_{(2)}\right), \\
\text { (resp. ad } \left.: A^{*} \otimes A^{*} \mapsto A^{*}: x \otimes y \mapsto S\left(y_{(1)}\right) x y_{(2)}\right) .
\end{gathered}
$$

The left and right adjoint actions of $A^{*}$ on itself are also called the left and right quantum dressing actions in quantum group theory (compare with Definition 2.5). By Theorem 3.10, the left (resp. right) adjoint action of $A^{*}$ on itself is a quantization of the right (resp. left) dressing action of $G$ on $G^{*}$. 
The proof of the following proposition follows immediately from the definition of the action $D^{\Phi}$. At the semi-classical level, it corresponds to the fact that a Poisson action on a Poisson manifold $P$ with a moment leaves symplectic leaves of $P$ invariant.

Proposition 3.12. The action $D^{\Phi}$ of $A^{*}$ on $V$ leaves every two-sided ideal of $V$ invariant.

Proposition 3.13. The map $\Phi: A^{*} \rightarrow V$ is $A^{*}$-equivariant with respect to the left adjoint action of $A^{*}$ on $A^{*}$ and the left action $D^{\Phi}$ of $A^{*}$ on $V$.

Proof. Let $x, y \in A^{*}, v \in V$. Then

$$
\Phi\left(\operatorname{ad}_{x} y\right)=\Phi\left(x_{(1)} y S\left(x_{(2)}\right)\right)=\Phi\left(x_{(1)}\right) \Phi(y) \Phi\left(S\left(x_{(2)}\right)\right)=x(\Phi(y)) .
$$

This proves the statement.

Q.E.D.

\section{Quantization of Semi-Direct Product Poisson Structures}

In this section, we give an example where quantum moment maps and reduction appear naturally. This example is also of its own interest, because it contains as a special case the Heisenberg double of a Hopf algebra as constructed by SemenovTian-Shansky in [STS2].

We start from the semi-classical picture. It is proved in [Lu1] that associated to each right Poisson action $\sigma: P \times G \rightarrow P$ of a Poisson group $G$ on a Poisson manifold $P$ there is a so-called semi-direct product Poisson structure on the manifold $P \times G^{*}$, where $G^{*}$ is the dual group of $G$, which encodes the Poisson structures on both $P$ and $G^{*}$, as well as the Poisson action $\sigma$ of $G$ on $P$. Denote by $\pi_{\mathrm{G}^{*}}$ and $\pi_{\mathrm{P}}$ the Poisson structures on $G^{*}$ and on $P$ respectively, and for $X \in \mathfrak{g}$, denote by $\sigma_{X}$ the infinitestimal generator of the action $\sigma$ in the direction of $X$. The semi-direct product Poisson structure on $P \times G^{*}$ is described in the following proposition.

Proposition 4.1. The following bi-vector field $\pi_{\sigma}$ defines a Poisson structure on the manifold $P \times G^{*}:$ for $p \in P, u \in G^{*}, \theta_{p}, \theta_{p}^{\prime} \in T_{p} P$ and $\theta_{u}, \theta_{u}^{\prime} \in T_{u}^{*} G^{*}$,

$$
\begin{aligned}
\pi_{\sigma}(p, u)\left(\left(\theta_{p}, \theta_{u}\right),\left(\theta_{p}^{\prime}, \theta_{u}^{\prime}\right)\right)= & \pi_{\mathrm{P}}(p)\left(\theta_{p}, \theta_{p}^{\prime}\right)+\pi_{G^{*}}(u)\left(\theta_{u}, \theta_{u}^{\prime}\right) \\
& +\left\langle\sigma_{r_{u}^{*} \theta_{u}}, \theta_{p}^{\prime}\right\rangle-\left\langle\sigma_{r_{u}^{*} \theta_{u}^{\prime}}, \theta_{p}\right\rangle,
\end{aligned}
$$

where $r_{u}^{*} \theta_{u} \in \mathfrak{g}$ is the right translate of $\theta_{u} \in T_{u}^{*} G^{*}$.

In the case when $P$ is the group $G$ itself and when the action $\sigma$ is the right action of $G$ on itself by right translations, we get a Poisson structure on the manifold $G \times G^{*}$. We will denote it by $\pi_{+}$.

We list some properties of the Poisson structure $\pi_{\sigma}$ in the following proposition. See [Lu2] and [Lu1] for proofs.

Proposition 4.2. 1) With the semi-direct Poisson structure $\pi_{\sigma}$ on $P \times G^{*}$, the projections from $P \times G^{*}$ to the two factors $P$ and $G^{*}$ are both Poisson maps.

2) Let $e \in G^{*}$ be the identity element of $G^{*}$. Then $P \subseteq P \times G^{*}: p \mapsto(p, e)$ is a coisotropic submanifold of $\left(P \times G^{*}, \pi_{\sigma}\right)$. 
3) The projection $P \times G^{*} \rightarrow G^{*}:(p, u) \mapsto u$ induces a right Poisson action of $G$ on $P \times G^{*}$. It is given by

$$
g:(p, u) \mapsto\left(\sigma(p, g), u^{g}\right),
$$

where $(u, g) \mapsto u^{g}$ is the right dressing action of $G$ on $G^{*}[L u-W e 1]$. In other words, it is the diagonal action of $\sigma$ and the right dressing action of $G$ on $G^{*}$.

4) The Poisson structure can be obtained as reduction by the diagonal subgroup of the action of $\left(G \times G, \pi_{\mathrm{G}} \oplus\left(-\pi_{\mathrm{G}}\right)\right)$ on $\left(P \times\left(G \times G^{*}\right), \pi_{\mathrm{P}} \oplus \pi_{+}\right)$given by

$$
G \times G \ni\left(h_{1}, h_{2}\right):(x, g, u) \mapsto\left(x \cdot h_{1}, h_{2}^{-1} g, u\right) .
$$

As a result, it is the unique Poisson structure on $P \times G^{*}$ such that the map

$$
p:\left(P \times\left(G \times G^{*}\right), \pi_{\mathrm{P}} \oplus \pi_{+}\right) \mapsto\left(P \times G^{*}, \pi_{\sigma}\right):(p, g, u) \mapsto(p \cdot g, u)
$$

is a Poisson map.

We now give a construction of a quantization of the semi-direct product Poisson structure $\pi_{\sigma}$ on $P \times G^{*}$, provided that the Poisson Lie group $G$, the Poisson manifold $P$ and the Poisson action $\sigma$ of $G$ on $P$ can be appropriately quantized. In fact, this will be the well-known construction in Hopf algebra theory, the so-called crossed product construction.

Let $A=\operatorname{Fun}_{q}(G)$ be the quantization of $G$ with $A^{*}=U_{q}(\mathfrak{g})$. Assume that $A_{\mathrm{P}}=\operatorname{Fun}_{q}(P)$ is a quantization of the Poisson structure on $P$ and that the right Poisson action $\sigma: P \times G \rightarrow P$ is quantized to a co-module map, also denoted by $\sigma$ :

$$
\sigma: A_{\mathrm{P}} \rightarrow A_{\mathrm{P}} \otimes A,
$$

making $A_{\mathrm{P}}$ into a right $A$-co-module algebra. Then the following map defines a left $A^{*}$-module structure on $A_{\mathrm{p}}$ :

$$
\Sigma: A^{*} \otimes A_{\mathrm{P}} \rightarrow A_{\mathrm{P}}: x \otimes f \mapsto x(f):=f_{(1)}\left\langle a_{(2)}, x\right\rangle,
$$

where $x \in A^{*}, f \in A_{\mathrm{P}}$ and $\sigma(f)=f_{(1)} \otimes a_{(2)} \in A_{\mathrm{P}} \otimes A$. Under this action, $A_{\mathrm{P}}$ becomes a left $A^{*}$-module algebra. We will also denote the operator $f \mapsto x(f)$ by $\Sigma_{x}$ for $x \in A^{*}$.

Consider now the two types of operators on $A_{\mathrm{p}}$ : the operators $\Sigma_{x}, x \in A^{*}$ and the multiplication operators $\mu_{f}: g \mapsto f g, f, g \in A_{\mathrm{P}}$. The fact that

$$
x\left(f_{1} f_{2}\right)=x_{(1)}\left(f_{1}\right) x_{(2)}\left(f_{2}\right)
$$

implies the following commutation relations between these two types of operators:

$$
\Sigma_{x} \mu_{f}=\mu_{x_{(1)}(f)} \Sigma_{x(2)} .
$$

This suggests that following definition of the algebra $A_{\mathrm{P}} \#{ }_{\sigma} A^{*}$, which we will later show to be a quantization of the semi-direct product Poisson structure $\pi_{\sigma}$.

Definition 4.3 ([Sw] [B-C-M]). As vector spaces, $A_{\mathrm{P}} \#{ }_{\sigma} A^{*} \cong A_{\mathrm{P}} \otimes A^{*}$, but we use $f \# x$ rather than $f \otimes x$, where $f \in A_{\mathrm{P}}$ and $x \in A^{*}$, to denote its elements. Multiplication in $A_{\mathrm{P}} \#_{\sigma} A^{*}$ is defined by the formula

$$
(f \# x)(g \# y)=f x_{(1)}(g) \# x_{(2)} y,
$$

where $f, g \in A_{\mathrm{P}}$ and $x, y \in A^{*}$. The fact that this defines an associative algebra

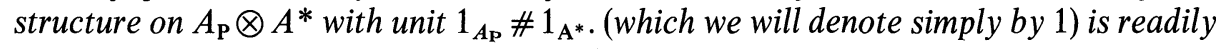


verified. The algebra $A_{\mathrm{P}} \#_{\sigma} A^{*}$ is called the semi-direct or $\mathbf{s m a s h}$ or crossed product of $A_{\mathrm{P}}$ and $A^{*}$ with respect to $\Sigma$.

Note that via the embeddings $A_{\mathrm{P}} \ni f \mapsto f \# 1_{\mathrm{A}^{*}}$ and $A^{*} \ni x \mapsto 1_{A_{\mathrm{P}}} \# x$, the algebras $A_{\mathrm{P}}$ and $A^{*}$ are naturally subalgebras of $A_{\mathrm{P}} \#_{\sigma} A^{*}$. From the discussion above, it is clear that the map

$$
A_{\mathrm{P}} \#{ }_{\sigma} A^{*} \rightarrow \operatorname{End}\left(A_{\mathrm{P}}\right): f \# x \mapsto \mu_{f} \Sigma_{x}
$$

defines a left $A_{\mathrm{P}} \#{ }_{\sigma} A^{*}$-module structure on $A_{\mathrm{P}}$.

Theorem 4.4. The algebra $A_{\mathrm{P}} \#_{\sigma} A^{*}$ is a quantization of the Poisson structure $\pi_{\sigma}$ on $P \times G^{*}$.

Proof. From the definition of the Poisson structure $\pi_{\sigma}$, the Poisson bracket between $x \in \operatorname{Fun}\left(G^{*}\right)$ and $f \in \operatorname{Fun}(P)$ is given by

$$
\{x, f\}(p, u)=\left(\sigma_{r_{u}^{*} d x(u)}(f)\right)(p), \quad p \in P, u \in G^{*} .
$$

Now consider $x$ and $f$ as in $A_{\mathrm{P}} \#{ }_{\sigma} A^{*}$. It suffices to show that

$$
\lim _{h \rightarrow 0} \frac{1}{h}(x f-f x)=\{x, f\} .
$$

Since the pairing between $A$ and $A^{*}$ satisfies

$$
\langle a, x\rangle=\varepsilon(a) \varepsilon(x)+h\langle d a(e), d x(e)\rangle+\cdots, \quad a \in A, x \in A^{*},
$$

where $e$ denotes the identity elements in both $G$ and $G^{*}$, the action $\Sigma$ of $A^{*}$ on $A_{\mathrm{P}}$ has the following expansion with respect to $h$ :

$$
\begin{aligned}
\Sigma_{x}(f) & =f_{(1)}\left\langle a_{(2)}, x\right\rangle \\
& =f_{(1)} \varepsilon(x) \varepsilon\left(a_{(2)}\right)+h f_{(1)}\left\langle d a_{(2)}(e), d x(e)\right\rangle \pm \cdots \\
& =\varepsilon(x) f+h \sigma_{d x(e)}(f)+\cdots
\end{aligned}
$$

Therefore,

$$
\begin{aligned}
x f & =\Sigma_{x_{(1)}} f \# x_{(2)} \\
& =\varepsilon\left(x_{(1)}\right) f \# x_{(2)}+h \sigma_{d x_{(1)}(e)}(f) \# x_{(2)}+\cdots \\
& =f x+h \sigma_{d x_{(1)}(e)}(f) \# x_{(2)}+\cdots .
\end{aligned}
$$

Now $x_{(2)} d x_{(1)}(e)$ is a $g$-valued function on $G^{*}$ given by $u \mapsto r_{u}^{*} d x(u)$. Hence

$$
\left(\sigma_{d x_{(1)}(e)}(f) \# x_{(2)}\right)(p, u)=\sigma_{r_{u}^{*} d x(u)}(f)(p)=\{x, f\}(p, u) .
$$

Therefore,

$$
\lim _{h \rightarrow 0} \frac{1}{h}(x f-f x)=\{x, f\} .
$$

This shows that the algebra $A_{\mathrm{P}} \#{ }_{\sigma} A^{*}$ is a quantization of the Poisson structure $\pi_{\sigma}$ on $P \times G^{*}$.

Q.E.D.

We now study the quantum analogs of the properties of $\pi_{\sigma}$ as stated in Proposition 4.2. 
First, the fact that the projections from $\left(P \times G^{*}, \pi_{\sigma}\right)$ to the two factors $P$ and $G^{*}$ are both Poisson obviously correspond to the fact that the embeddings

$$
A_{\mathrm{P}} \rightarrow A_{\mathrm{P}} \#{ }_{\sigma} A^{*}: f \mapsto f \# 1_{\mathrm{A}^{*}} .
$$

and

$$
A^{*} \rightarrow A_{\mathrm{P}} \#{ }_{\sigma} A^{*}: x \mapsto 1_{A_{\mathrm{P}}} \# x
$$

are algebra homomorphisms.

Secondly, the coisotropic embedding of $P$ into $P \times G^{*}$ given by $p \mapsto(p, e)$ can be described by the kernel of the map

$$
\operatorname{Fun}(P) \otimes \operatorname{Fun}\left(G^{*}\right) \rightarrow \operatorname{Fun}(P): f \otimes x \mapsto x(e) f .
$$

According to the "Poisson Creed" stated earlier, its quantum counterpart should be a left ideal of the algebra $A_{\mathrm{P}} \#_{\sigma} A^{*}$.

Proposition 4.5. The kernel of the map

$$
\lambda: A_{\mathrm{P}} \#_{\sigma} A^{*} \rightarrow A_{\mathrm{P}}: f \# x \mapsto \varepsilon(x) f,
$$

is a left ideal of the algebra $A_{\mathrm{P}} \#_{\sigma} A^{*}$.

Proof. The statement can be proved directly from the definition: if $\lambda(f \# x)=\varepsilon(x) f=0$, then for any $g \# y \in A_{\mathrm{P}} \#_{\sigma} A^{*}$, we have

$$
\begin{aligned}
\lambda((g \# y)(f \# x)) & =\lambda\left(g y_{(1)}(f) \# y_{(2)} x\right) \\
& =\varepsilon\left(y_{(2)}\right) \varepsilon(x) g y_{(1)}(f) \\
& =g y(\varepsilon(x) f) \\
& =0 .
\end{aligned}
$$

This shows that the kernel of the map $\lambda$ is a left ideal of the algebra $A_{\mathrm{P}} \#{ }_{\sigma} A^{*}$.

A more illustrating proof is by considering the representation of $A_{\mathrm{P}} \#_{\sigma} A^{*}$ on $A_{\mathrm{P}}$ given by

$$
A_{\mathrm{P}} \#{ }_{\sigma} A^{*} \ni f \# x \mapsto \mu_{f} \Sigma_{x} .
$$

Since $x\left(1_{A_{\mathrm{p}}}\right)=\varepsilon(x) 1_{A_{\mathrm{p}}}$, we have

$$
\lambda(f \# x)=(f \# x)\left(1_{A_{\mathrm{p}}}\right) .
$$

Consequently, the kernel of $\lambda$ consists of exactly the annihilators of the element $1_{A_{\mathrm{p}}}$ in $A_{\mathrm{P}} \#_{\sigma} A^{*}$, and it is thus a left ideal of $A_{\mathrm{P}} \#_{\sigma} A^{*}$.

Q.E.D.

Corresponding to 3) of Proposition 4.2, we have

Proposition 4.6. The left $A^{*}$-module algebra structure on $A_{\mathrm{P}} \#_{\sigma} A^{*}$ with moment map

$$
A^{*} \rightarrow A_{\mathrm{P}} \#{ }_{\sigma} A^{*}: x \mapsto 1 \# x,
$$

where 1 denotes the identity element of $A_{\mathrm{P}}$, is given by the following (diagonal) action:

$$
x: f \# y \mapsto x_{(1)}(f) \# \operatorname{ad}_{x_{(2)}}^{l}(y), \quad x, y \in A^{*}, f \in A_{\mathrm{P}} \#_{\sigma} A^{*},
$$

where $\mathrm{ad}^{l}$ denotes the left adjoint action of $A^{*}$ on itself. By Theorem 3.10, it is a quantization of the right dressing action of $G$ on $G^{*}$. 
Proof. By Definition 3.9, the left action of $A^{*}$ on $A_{\mathrm{P}} \#_{\sigma} A^{*}$ induced by the map $x \mapsto 1 \# x$ is given by

$$
\begin{aligned}
x(f \# y) & =\left(1 \# x_{(1)}\right)(f \# y)\left(1 \# S\left(x_{(2)}\right)\right) \\
& =x_{(1)(1)}(f) \# x_{(1)(2)} y S\left(x_{(2)}\right),
\end{aligned}
$$

where

$$
\Delta x_{(1)}=x_{(1)(1)} \otimes x_{(1)(2)}
$$

Let

$$
\Delta x_{(2)}=x_{(2)(1)} \otimes x_{(2)(2)} .
$$

By the co-associativity of $\Delta$, we have

$$
x_{(1)(1)} \otimes x_{(1)(2)} \otimes x_{(2)}=x_{(1)} \otimes x_{(2)(1)} \otimes x_{(2)(2)} .
$$

Therefore,

$$
\begin{aligned}
x(f \# y) & =x_{(1)}(f) \# x_{(2)(1)} y S\left(x_{(2)(2)}\right) \\
& =x_{(1)}(f) \# \operatorname{ad}_{x_{(2)}}^{l} y .
\end{aligned}
$$

Before studying the quantum analog of 4) in Proposition 4.2, we first introduce the following definition.

Definition 4.7. The semi-direct product algebra $A \#_{\Delta} A^{*}$, where $\Delta: A \rightarrow A \otimes A$ is the co-product of $A$, considered as defining a right $A$-co-module structure on $A$, is called the Heisenberg Double of $A$ [STS2]. By Theorem 3.10, it is a quantization of the Poisson structure $\pi_{+}$, the semi-direct product Poisson structure on $G \times G^{*}$ defined by the right action of $G$ on itself by right translations.

Semi-classically, the action of $G$ on $\left(G \times G^{*}, \pi_{+}\right)$given by

$$
g:(h, u) \mapsto(g h, u)
$$

is a Poisson action. The following proposition of the quantum analog of this action can be proved directly from the definition.

Proposition 4.8. The map

$$
(\Delta \otimes \mathrm{id}): A \#_{\Delta} A^{*} \rightarrow A \otimes\left(A \#_{\Delta} A^{*}\right)
$$

makes $A \#_{\triangle} A^{*}$ into a left $A$-co-module algebra.

Consider now the left action of $A^{*}$ on $A_{\mathrm{P}}$ and the right action of $A^{*}$ on $A \#{ }_{\Delta} A^{*}$ given by

$$
A^{*} \ni x: a \# y \mapsto\left\langle x, a_{(1)}\right\rangle a_{(2)} \# y .
$$

The latter is the right action of $A^{*}$ on $A \#_{\Delta} A^{*}$ induced by $\Delta \otimes$ id. These two actions together define a left action of $A^{*} \otimes A^{*}$ on $A_{\mathrm{P}} \otimes\left(A \#_{\Delta} A^{*}\right)$ :

$$
A^{*} \otimes A^{*} \ni x \otimes y: f \otimes(a \# z) \mapsto x(f) \otimes\left\langle S(y), a_{(1)}\right\rangle a_{(2)} \# z .
$$

This action makes the algebra $A_{\mathrm{P}} \otimes\left(A \#_{\Delta} A^{*}\right)$ into a left $A^{*} \otimes A^{*}$-module algebra but with the co-product on $A^{*} \otimes A^{*}$ being $\Delta \otimes \Delta^{\text {op }}$. As in the semi-classical case, the algebra $A_{\mathrm{P}} \#{ }_{\sigma} A^{*}$ can be obtained as reduction by the "diagonal" $J:=\Delta\left(A^{*}\right)$ of 
$A^{*} \otimes A^{*}$ (see Example 3.7). As a result, the algebra structure of $A_{\mathrm{P}} \#_{\sigma} A^{*}$ is the unique one on the vector space $A_{\mathrm{P}} \otimes A^{*}$ such that the map

$$
\sigma \otimes \mathrm{id}: A_{\mathrm{P}} \otimes A^{*} \rightarrow A_{\mathrm{P}} \otimes\left(A \#_{\Delta} A^{*}\right)
$$

is an algebra homomorphism.

Acknowledgement. The author would like to thank Viktor Ginzburg, Alan Weinstein, Ping Xu and Ilya Zakharevich for helpful discussions.

\section{References}

[B-C-M] Blattner, R.J., Cohen, M., Montgomery, S.: Crossed products and inner actions of Hopf algebras. Trans. Am. Math. Soc. 298, 671-711 (1986)

[Dr] Drinfel'd, V.G.: Hamiltonian structures on Lie groups, Lie bialgebras and the geometric meaning of the classical Yang-Baxter equations. Sov. Math. Dokl. 27 (1), 68-71 (1983)

[Ho] Howe, R.: Dual pairs in physics. Harmonic Oscillators, Photons, Electrons, and Singletons, Lectures in Applied Mathematics 21, 179-207 (1985)

[Lu-We1] Lu, J.H., Weinstein, A.: Poisson Lie groups, dressing transformations, and Bruhat decompositions. J. Diff. Geom. 31, 501-526 (1990)

[Lu-We2] Lu, J.H., Weinstein, A.: Groupoïdes symplectiques doubles des groupes de LiePoisson. C. R. Acad. Sc. Paris 309, 951-954 (1989)

[Lu1] Lu, J.H.: Momentum mappings and reductions of Poisson Lie group actions. Proceedings of the Seminaire Sud-Rhodanien de Geometrie à Berkeley, 1989, 1991 Springer-MSRI series

[Lu2] Lu, J.H., Multiplicative and affine Poisson structures on Lie groups. PhD thesis, University of California, Berkeley (1990)

[Mj] Majid, S.: Quasitriangular Hopf algebras and Yang-Baxter equations. Int. J. Mod. Phys. A 5 (1), 1-91 (1990)

[Ms-We] Marsden, J., Weinstein, A.: Reduction of symplectic manifolds with symmetry. Rep. Math. Phys. 5, 121-129 (1974)

[R-T-F] Reshetikhin, N.Y., Takhtajan, L.A., and Faddeev, L.D.: Quantization of Lie groups and Lie algebras. Leningrad Math. J. 1, 193-225 (1990)

[STS1] Semenov-Tian-Shansky, M.A.: Dressing transformations and Poisson Lie group actions. Publ. RIMS, Kyoto University 21, 1237-1260 (1985)

[STS2] Semenov-Tian-Shansky, M.A.: Poisson Lie groups, quantum duality principle and twisted quantum double. Preprint (1992) (in Russian)

[Sw] Sweedler, M.E.: Hopf algebras. New York: Benjamin (1969)

[We1] Weinstein, A.: The symplectic category. Lecture Notes in Math. 905 (1982)

[We2] Weinstein, A.: Coisotropic calculus and Poisson groupoids. J. Math. Soc. Japan 40 (4), 705-727 (1988)

Communicated by N.Yu. Reshetikhin 